\title{
EchoGéo
}

$9 \mid 2009$

L'Asie centrale : le temps des recompositions

\section{Entretien avec Yvette Dewolf}

\author{
Etienne Cossart
}

\section{OpenEdition}

Journals

Édition électronique

URL : https://journals.openedition.org/echogeo/11274

DOI : 10.4000/echogeo. 11274

ISSN : 1963-1197

\section{Éditeur}

Pôle de recherche pour l'organisation et la diffusion de l'information géographique (CNRS UMR 8586)

\section{Référence électronique}

Etienne Cossart, « Entretien avec Yvette Dewolf », EchoGéo [En ligne], 9 | 2009, mis en ligne le 26 juin 2009, consulté le 31 juillet 2021. URL : http://journals.openedition.org/echogeo/11274 ; DOI : https:// doi.org/10.4000/echogeo. 11274

Ce document a été généré automatiquement le 31 juillet 2021.

EchoGéo est mis à disposition selon les termes de la licence Creative Commons Attribution - Pas d'Utilisation Commerciale - Pas de Modification 4.0 International (CC BY-NC-ND) 


\title{
Entretien avec Yvette Dewolf
}

\author{
Etienne Cossart
}

1 Les formations superficielles constituent un enjeu primordial pour l'Homme: en recouvrant d'un manteau quasiment continu l'ensemble des terres émergées, elles constituent un support pour les sociétés ou même, plus largement, du monde vivant. De leur diversité dépendent un grand nombre de ressources et de contraintes avec lesquelles les sociétés doivent composer. En cela, les "formations superficielles " sont un objet d'étude central pour le géographe. Pourtant, la richesse d'un tel patrimoine reste mal connue, d'approche fragmentaire.

2 Nous comprenons dès lors tout l'intérêt de l'ouvrage, aussi ambitieux que riche, proposé et dirigé par Yvette Dewolf et

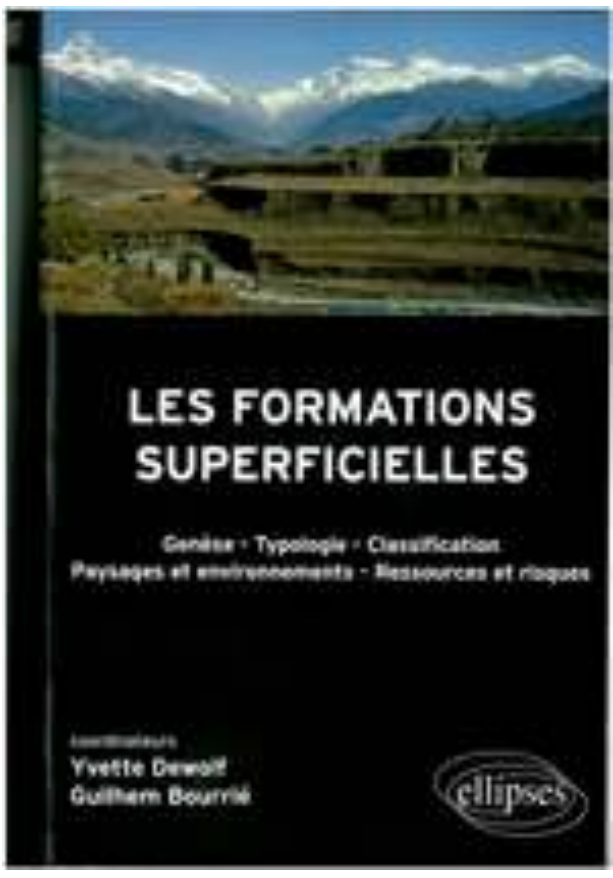
Guilhem Bourrié. Il réunit les contributions de plus de cinquante auteurs provenant tant de disciplines différentes (géomorphologues, pédologues, géologues, ingénierie de l'environnement, etc.) que d'institutions différentes (universités, centres de recherches, bureaux d'étude).

3 L'ouvrage permet ainsi de montrer comment les formations superficielles sont corrélatives du façonnement du relief et donc comment elles permettent de retracer l'histoire des milieux, de comprendre les liens existant entre notre environnement et les climats actuels ou passés. Mais, au-delà de ces aspects qui peuvent s'apparenter à une recherche dite «fondamentale », l'ouvrage est résolument appliqué. Les méthodes et techniques permettant d'étudier les formations superficielles sont ainsi détaillées, en laissant bien sûr une large part au travail de terrain et à la restitution des observations 
de terrain par des documents cartographiques. De multiples exemples montrent les ressources offertes par les matériaux que constituent les formations superficielles (sols, granulats, etc.), mais également les risques inhérents à ces formations. En effet, de leurs caractéristiques (granulométriques, mécaniques) dépendent la localisation de glissements de terrain, l'extension d'inondations ou la propagation de pollutions.

4 Au final, cet ouvrage réunit les contributions pointues de spécialistes, tout en étant accessible à un très large public: la richesse des illustrations, combinée aux efforts pour trouver une ligne éditoriale pédagogique, en facilite grandement la lecture. Nul doute qu'un large public d'étudiants, de chercheurs, de praticiens de l'environnement pourra se référer utilement à cet ouvrage majeur de la géographie physique et environnementale francophone.

Échogéo

Vous présentez un ouvrage impressionnant par son volume et sa qualité : quelle est l'origine de cet ouvrage ? D'où en est venue l'idée?

\section{Yvette Dewolf}

Le fondement du problème est : «qu'est-ce qu'une formation superficielle? ». Le terme de «sol » a longtemps été employé, mais laissons le aux pédologues! Les formations superficielles, en étant corrélatives de l'évolution du relief, permettent d'appréhender la dynamique des milieux. Or la compréhension de cette dynamique, fondée à la fois sur le terrain et la cartographie, est le gage d'une bonne gestion des milieux : il fallait donc réunir en un seul ouvrage des approches variées et complémentaires, du terrain au laboratoire.

Échogéo

Près de 800 pages, 60 planches couleurs, de multiples tableaux et croquis : n'a-t-il pas été difficile de trouver un éditeur?

\section{Yvette Dewolf}

L'idée de l'ouvrage a d'emblée plu à l'éditeur «Ellipses » : ils ont compris qu'il y avait un manque pour un très large public de chercheurs, d'enseignants, d'étudiants, mais aussi de forestiers, d'archéologues, d'ingénieurs. Etant donné le volume de l'ouvrage, nous souhaitions initialement faciliter sa manipulation en le subdivisant en quatre tomes. Néanmoins, Ellipses nous a incité à conserver jusqu'au bout, même dans l'impression, l'unité de l'ouvrage, son fil conducteur.

Échogéo

Le collectif réunit plus de cinquante spécialistes aux champs de compétences complémentaires (géomorphologie, géologie, pédologie, cartographie et télédétection, etc.). Comment fut-il constitué?

\section{Yvette Dewolf}

Très facilement! On connaissait presque tout le monde et dès les premières réunions collectives, à l'institut de géographie, l'ensemble du collectif a été très enthousiaste. Autour d'un premier noyau d'auteurs, quelques contributeurs supplémentaires sont ensuite entrés en contact avec moi afin de participer à ce projet. En fait la plus grande difficulté a été d'homogénéiser les niveaux entre les contributions : certains auteurs rédigeaient des chapitres correspondant à des articles dignes des meilleures revues internationales, d'autres se contentaient d'un cours de premier cycle... Des relectures multiples et attentives par Pierre Freytet et Fernand Joly ont permis de trouver le ton 
le plus juste possible dans les différents chapitres. Ce processus a bien sûr nécessité un certain temps... Il faut enfin mentionner le travail considérable effectué par Eliane Leterrier, qui a redessiné l'ensemble des figures afin de leur conférer une homogénéité graphique : cela contribue grandement à l'unité de l'ouvrage. discussions avec Fernand Joly sur les méthodes de cartographie : le choix des couleurs, les possibilités ou impossibilités techniques. Dans le cadre du laboratoire de géomorphologie de Caen, nous avons réfléchi pour créer quelque chose de nouveau, en représentant les formations superficielles sur les cartes. Jean Tricart proposait une représentation chronologique des formations superficielles, nous tenions davantage à une représentation morphodynamique : de là est apparue la cartographie actuelle. Il est d'ailleurs intéressant de voir, aujourd'hui, les géologues à la recherche de cartographes des formations superficielles : un poste a même été récemment publié au BRGM. Quand je vois cela, je me dis : on a gagné !

29 Échogéo

30 Cependant, on voit bien aujourd'hui que l'accès aisé à de multiples images, restituant des terrains même lointains et difficiles d'accès, incite des chercheurs à économiser le 
travail de rendu cartographique, voire le travail de terrain lui-même. Qu'en pensezvous? comment est née une formation superficielle, pourquoi elle est là et comment elle va évoluer. En cela le travail du géomorphologue est essentiel : nous avons une vision spatiale et dynamique que les ingénieurs et les géologues n'ont pas.

\section{RÉSUMÉS}

L'entretien porte sur l'ouvrage d'Yvette Dewolf et Guilhem Bourrié, Les formations superficielles, Genèse, Typologie, Classification, Paysages et environnements, Ressources et risques. Paris, Ellipses, 2008, 798 p.

\section{AUTEUR}

\section{ETIENNE COSSART}

Etienne Cossart (etienne.cossart@univ-paris1.fr) Université Paris 1- Panthéon Sorbonne et UMR CNRS PRODIG 8586) 